\title{
Charge Exchange Recombination Spectroscopy on a Diagnostic Hydrogen Beam - Measuring Impurity Rotation and Radial Electric Field at the Tokamak TEXTOR
}

\author{
J.W.Coenen,M.Clever, U.Samm, O.Schmitz, B.Schweer, \\ S. Freutel, H.Stoschus, B.Unterberg and the TEXTOR Team \\ Institut für Energieforschung - IEF-4: Plasmaphysik, Forschungszentrum Jülich \\ GmbH, Assoziaton EURATOM-FZJ, Partner in the Trilateral Euregio Cluster, 52425 \\ Juelich, Germany
}

\begin{abstract}
In this work we present an overview on the CXRS diagnostic operated with the modulated diagnostic hydrogen beam at the Tokamak TEXTOR. The diagnostic setup combines two observation systems used for the measurement of the poloidal $\left(v_{\text {pol }}\right)$ and the toroidal $\left(v_{\text {tor }}\right)$ ion velocity component.

At TEXTOR a differential Doppler spectroscopy approach (accurate absolute rotation scale) is combined with the high intensity and spatial resolution of a direct imaging system necessary for accurate poloidal rotation measurements on a shot by shot basis. This setup allows the full utilization of the a $2 \mathrm{D}$ CCD detector in the spectral and radial direction. In case of the poloidal system this allows spatial resolution in the range of $\mathrm{mm}$ to $\mathrm{cm}$ depending on the intensity requirements for the velocity. The toroidal system is comprised of a fiber-optic array. The combination of the two measurements with a low power diagnostic beam can in principal be operated during any available heating scenario without interfering with the discharge. Time resolution is limited by the necessary averaging process, typically a stable plateau of $3 \mathrm{~s}$ during a TETXOR pulse is used. The TEXTOR tokamak has the ability to apply momentum input with two tangential neutral beam heating injectors, allowing for measurements under various heating and momentum input scenarios. With the presented diagnostic half the plasma minor radius at a spatial resolution of $\sim 1 \mathrm{~cm}$ is covered. With the CVI line at $529.053 \mathrm{~nm}$ an accuracy of $0.7 \mathrm{~km} / \mathrm{s}$ for the poloidal and $\sim 5 \mathrm{~km} / \mathrm{s}$ for the toroidal system is given. The temperature is measured with an accuracy of a few $\mathrm{eV}$. The presented work illustrates the capability of the system during a toroidal momentum scan, showing the self-consistent determination of the radial electric field from experimental CXRS data based on the radial force balance.
\end{abstract}




\section{Introduction}

In fusion experiments the properties of magnetic confinement largely depend on the impurity concentration, as well as on the plasma rotation and the radial electric field. Rotation can influence screening of external perturbations, suppress the onset of modes [8] and in case of strong rotation shear can lead to a suppression of turbulent transport. Charge exchange spectroscopy (CXRS) (cf. $[39,14])$ is the most successful tool for the measurement of impurity rotation and ion temperature $\left(T_{i}\right)$ profiles $[17,25]$. The radial electric field $\left(E_{r}\right)[24]$ is known to be connected to transport changes in the plasma edge, including the L-H transition([40,35]). Knowing this, it is important to study the $E_{r}$ and its connection to transport and confinement.

At TEXTOR two independent CXRS diagnostics are employed as is depicted in figure 1. The first makes use of a 1.2 MW Neutral Heating Beam and provides fast routine measurements of $T_{i}, v_{\text {tor }}$ and $n_{i}$, with a time resolution of a few milliseconds, but a poor spatial resolution of the order of 40 to $80 \mathrm{~mm}$. [41] The second system, being presented here utilizes a dedicated low-power hydrogen diagnostic beam [9] allowing high spatial resolution, typically 10 to $20 \mathrm{~mm}$ on a shot by shot basis. Typical TEXTOR plasma have a plateau phase of several seconds allowing averaging without compromising the measurement. It is used to consistently study the $E_{r}$, measuring the $\operatorname{poloidal}\left(v_{p o l}\right)$ and toroidal rotation $\left(v_{t o r}\right)$, as well as the ion temperature $\left(T_{i}\right)$ and density. The $E_{r}$ is based on the radial force balance (Equation (5)). The spectroscopic measurements are

Figure 1: (ONLINE COLOR)(left) $\operatorname{TEXTOR}(\mathrm{R}=1.75 \mathrm{~m} \mathrm{r}=46 \mathrm{~cm})$ and diagnostics locations. (right) CXRS Diagnostic setup mounted at the Low Field Side.

used to obtain the rotation via Doppler shift spectroscopy from the spectral position with respect to an unshifted reference, while the temperature measurements are based on the broadening of the spectra. The carbon density can be calculated based on the intensity. The speciality for the $v_{\text {pol }}$ measurement at TEXTOR is the intrinsic calibration via opposing lines of sight, differential Doppler spectroscopy (Section 3.1). All measurements are performed on the $C V I$ line $(529 \mathrm{~nm})$ CXRS line. The main focus here lies on the ability to measure $E_{r}$ over half the minor radius $(0.5<r / a<1.1)$ consistently without any external input needed. All components of the radial force balance (cf. Equation (5)) can be measured simultaneously at one toroidal position of the tokamak for any heating scenario. Here the CXRS setup is presented which has been developed at TEXTOR $[28,2,5] . v_{p o l}, v_{t o r}, T_{i}$ and $n_{i}$ can be measured using the hydrogen beam RuDI [26, 9, 30]. Systems applying similar diagnostic beams are operated TJII stellarator [4] and TCV [27, 11]

The paper is organized as follows. In the first part of this paper the design parameters of the complete system are given. In the second part the measurement principles for $v, T, n$ are described, including the use of beam modulation for background substraction, the 
principle of differential Doppler shift spectroscopy and the methods to obtain a radial calibration (Section 3.1) via direct measurement or beam emission spectroscopy (BES). In the third and last part, an assessment of the accuracy is given as well as the principles for the error bar calculations which is followed by a consistency check on exemplary data.

\section{Diagnostic Setup}

In order to survey the challenges for accurate measurements with the CXRS diagnostic we introduce the boundary conditions set by the TEXTOR plasma and the setup itself. The resolution in $v_{p o l}$ and $v_{t o r}$ is required to be $<1 \mathrm{~km} / \mathrm{s}$ and $<10 \mathrm{~km} / \mathrm{s}$ respectively based on measurements at TEXTOR and comparable experiments[2, 11$]$. A high spectral resolution instrument with a dispersion of $1 \mathrm{pm} /$ pixel for $v_{\text {pol }}$ is required. For such small bandwidth the signal intensities might be strongly interfered by photon statistics that limits the achievable spectral resolution (Section4). The used hydrogen beam delivers, in contrast to large heating NBI devices [3], only a small equivalent current, which leads to a small emission of charge exchange light and hence making intensity one of main diagnostic challenges (cf. Section 4). The typical ion current in the source of $\sim 2$ A leads to a neutral particle current of about $1.2 \mathrm{~A}$ at $50 \mathrm{kV}$ acceleration voltage, with a width $(1 / \mathrm{e})$ inside the vessel of $8 \mathrm{~cm}$ at $R=1.75 \mathrm{~m}\left( \pm 0.5^{\circ}\right.$ divergence) [28]. The beam modulation $(10 \mathrm{~Hz})$ presents an easy approach to background subtraction as described in Section 3, differentiating between active charge exchange from the beam and passive charge exchange background. The low power input allows measurements during ohmic discharges without disturbing the plasma. In addition to the requirements for the rotation measurements the system should be capable of measuring the temperature over the whole temperature range accessible in the observed volume at TEXTOR $30 \mathrm{eV}<T_{i}<1000 \mathrm{eV}$ [38, 23]. Another set of measurements involves the influence of the DED [16] on the magnetic topology [37, 36]. To resolve this a spatial resolution between 1 and $2 \mathrm{~cm}$ is required. The typical averaging time for the measurement is typically 3 seconds in order to gather enough light for the high velocity resolution (cf. section 4). To assure accurate measurements the plateau of the discharge is used. The frame rate with which the data is acquired is $10 \mathrm{~Hz}$ due to the background subtraction sheme (cf. Section 3) based on beam modulation. Time resolved measurements $(\Delta t=100 \mathrm{~ms})$ can be performed when in turn accepting larger error-bars for the measured rotation values, and thus loosing the capability of resolving changes in the $v_{p o l}$ and the $E_{r}$ due to poisson statistics (cf. section 4) at low intensities. The diagnostic arrangement consists of three distinct systems, the hydrogen diagnostic beam, the toroidal and poloidal observations, including the subsequent spectrometers and camera DAQ systems. As is depicted in figure 1 the diagnostic beam is injected from the low field side of TEXTOR, while the observation systems are mounted nearly perpendicular to the direction of the beam in the poloidal and toroidal (equatorial) plane. In the following section each component shall be introduced, with a focus on the detailed description of the observation systems. Both systems cover half the minor 
radius of TEXTOR $(0.5<r / a<1.1)$ allowing coverage of the plasma edge (SOL) up to the plasma core. They are optimized to deliver as much intensity as possible to minimize the effects of photon statistics on the measured quantities (cf. section 4).

\subsection{Poloidal Observation System}

The poloidal system observes in the poloidal plane of the tokamak vessel, as depicted in figure 1. It is equipped with a direct imaging from the vessel top and an additional bundle (3 channels) of optical fibers with a line of sight from the bottom of the vessel (opposing lines of sight) for the differential Doppler spectroscopy. Mounting a camera close to the tokamak may in future be difficult considering energetic radiation and neutrons, in case of TEXTOR however here it is a feasible way to gain intensity. Figure 2 gives an overview of the properties of this poloidal observation system. The direct imaging is optimized in two ways keeping the available hardware

Figure 2: (ONLINE COLOR) Optical components, of the poloidal (Poloidal Cut) as well as the toroidal observation system and its optical elements (Top View)

constraints in mind: Firstly, the direct imaging components (figure 2) relay the $270 \mathrm{~mm}$ observation length onto the entrance slit $(\mathrm{h}=18 \mathrm{~mm}, \mathrm{w}=200 \mu \mathrm{m})$ (magnification $1: 15$ ) and subsequently through the spectrometer onto an image intensifier (fiber coupled with CCD) $(25 \mathrm{~mm} \equiv 10.5 \mathrm{~mm}$ Chip) and secondly the numerical aperture (NA) is optimized to satisfy as much as possible the limitations due to the spectrometer $(\mathrm{NA}=0.05$, table 1). Details for the spectrometer and camera system are given in section 2.3

The distance between the RuDI beam and the first optical element (L1) is $74 \mathrm{~cm}$. The in-vessel components (L1\& L2) were optimized for an image NA of 0.4 [2] and are imaging the emission through the window onto the relay optics (L3-L5). This total setup has a length of about $216 \mathrm{~cm}$. By using a direct imaging system instead of the old fiber optics based system almost an order of magnitude in intensity was gained due to improved numerical apertures. In addition a broadband anti reflection coating was applied for the range of 400 to $700 \mathrm{~nm}$.

The lower observation for the differential Doppler spectroscopy is located $99 \mathrm{~cm}$ away from the beam, with one (L4b) lens imaging the light onto the optical fibers $(\mathrm{NA}=0.4)$ and another one (L6) imaging the fibers onto the spectrometer slit via a small mirror. Both parts are inclined by an angle of about $11^{\circ}$ to the beam due to the available access points. This fact is used to perform beam emission spectroscopy (BES) to determine the radial position of the viewing lines via the Doppler shift. The radial channels can be

Figure 3: Spatial resolution for the poloidal and toroidal system using a channel spacing of $1 \mathrm{~cm}$ for the poloidal and actual viewing lines for the toroidal view. 
chosen depending on the experimental scenario and the intensity requirements. Since a CCD camera is used, it is possible to either readout each CCD line, hence realize a very high spatial resolution with low intensity, or combine some channels (binning) in order to have reasonable spatial resolution and a good intensity. The spatial or radial resolution is determined by the size of the observed beam volume. A channel observing $1 \mathrm{~cm}$ of the beam will collect light from the complete volume, defined by the incident angle and the beam width. The radial resolution is in the order of 1-2 cm (cf. figure 3 . The poloidal system has a specially good resolution in the plasma edge due to the small incident angle with the beam at larger minor radius. This is of special interest for edge transport studies, as well as the measurement of the $E_{r}$ in the edge. The resolution plotted in figure 3 represents an evenly spaced binning of the channels. Different radial resolutions for each part of the observation volume can be achieved via CCD binning. The overlap between several channels is only resolvable by sacrificing radial resolution.

\subsection{Toroidal Observation System}

The toroidal observation system is mounted in the equatorial plane of the tokamak observing the hydrogen diagnostic beam via a small mirror tangentially(figure $1 \& 2$ ). It consists of the collecting part (L1, L2) inside the tokamak vessel including the small mirror collecting the light from the direction of the beam, as well as a set of lenses to image onto the optical fibers (L3). The design was optimized to transfer the light to the fibers mounted at the image side. The fibers have a numerical aperture of 0.4 and a diameter of $1 \mathrm{~mm}$. In contrast to the poloidal system the radial spacing is given by the size and the mounting of the optical fibers. The core of the fibers is $0.8 \mathrm{~mm}$ and leads to an object size of $\sim 14 \mathrm{~mm}$ (magnification 1:17.5). The radial spacing and resolution is shown in figure 3 is comparable to the typical poloidal setup. The fibers are arranged in a block of $2 \times 10$ fibers, giving 2 fibers per radial channel (10 total), in order to improve the intensity available for each radial channel. In contrast to the poloidal system the resolution decreases to the plasma edge. Structural constraints inside the vessel leave this as the only viable viewing option for the toroidal observation system. In front of the spectrometer the fibers form a single line are imaged onto the $250 \mu \mathrm{m}$ wide spectrometer slit using a small relay optic (cf. Section 2.3. In between the first 5 channels (10 fibers) and the last 5 channels (10 fibers) an additional fiber is supplied for the neon reference $(\lambda=529.82 \mathrm{~nm})$ produced by Geissler discharge neon lamp [33, 19]. All fibers are mounted in a straight brass housing, supplying accuracy and support to assure all fibers are aligned perfectly parallel to the reference channel.

\subsection{Spectrometers \&5 Camera systems}

The light dispersion elements used in the actual setup are two high resolution spectrometers as described in the overview table 1.

High resolution is required to resolve the particularly small poloidal velocities of order $\mathrm{km} / \mathrm{s}$. The light collecting parts are optimized for high transmission since the 
spectrometers themselves have got very small numerical apertures (table 1) and etendue. The values are $0.021,0.072$ for the poloidal and toroidal system, respectively. This trade off between resolution and photon statistics is the limiting factor for the capabilities of this diagnostic (Section 4). Both spectrometers are mounted in Littrow configuration

\begin{tabular}{l|c|c} 
& Poloidal System & Toroidal System \\
\hline Spektrometer Type & Littrow & Littrow \\
Grating Type & Echelle & $(160 \times 130)\left[\mathrm{mm}^{2}\right]$ \\
\hline Grating Size & $(220 \times 110)\left[\mathrm{mm}^{2}\right]$ & $-/ 2$ \\
Line Density (Blaze) & $79.015\left[\frac{\text { Lines }}{\mathrm{mm}}\right]\left(76^{\circ}\right)$ & $1200\left[\frac{\text { Lines }}{\mathrm{mm}}\right]\left(57.37^{\circ}\right)$ \\
\hline Filter / Order & CVI $529 \mathrm{~nm} \pm 2 \mathrm{~nm} / 46$ & $-/ 2$ \\
\hline Focal Length $[\mathrm{mm}] /$ NA & $1000 / 0.05$ & $750 / 0.08$ \\
\hline Dispersion $\left[\frac{10^{-10} \mathrm{~m}}{\text { pixel }}\right]$ & $(10.799 \pm 0.003) 10^{-3}$ & $(0.1624 \pm 0.0005)$ \\
Observed $\lambda\left[10^{-10} \mathrm{~m}\right]$ & CVI 5290.53 & CVI 5290.53 \\
\hline Camera Type & AVT Pike F100B & DALSA CA D1 \\
Chipsize[pixel $\left.{ }^{2}\right]$ & $1000 \mathrm{x} 1000$ & $256 \mathrm{x} 256$ \\
\hline Quantum Efficiency (CCD @ 529nm) & $\sim 45 \%$ & $\sim 50 \%$ \\
Quantum Efficiency (MCP @ 529nm) & $\sim 10 \%$ & $\sim 12 \%$
\end{tabular}

Table 1: Parameters for both, the poloidal and toroidal, observation systems

with a blazed grating. Since intensity is of the essence both gratings are used near the blaze angle. The poloidal spectrometer with a focal length of $1 \mathrm{~m}$ and a high dispersion Echelle grating is optimized for the use at $\lambda=529.053 \mathrm{~nm}$ in the $46^{\text {th }}$ order, while the toroidal spectrometer is equipped with a holographic grating an operated in the $2^{\text {nd }}$ order. Due to the high order used for the poloidal system several other lines may be visible in the observation region. A bandpass filter with a width of $2 \mathrm{~nm}$ is used. The poloidal spectrometer in particular is optimized for use on the $C V I$ line but both spectrometers can cover most parts of the available spectral range $(400-700 \mathrm{~nm})[2,28]$. The width of the slit of the poloidal and toroidal spectrometer is $200 \mu \mathrm{m}$ and $250 \mu \mathrm{m}$, leading to an instrument function of 20 pixel and 12 pixel for the poloidal and toroidal system respectively after imaging on the subsequent cameras. The dispersion of the two spectrometers, measured with emissions from an argon (Ar I: $528.6 \mathrm{~nm}, 529.0 \mathrm{~nm}$ ) [31] and neon (Ne I: 532.1nm, 534.1nm, 534.3nm) [31] reference lamp around $\lambda=529 \mathrm{~nm}$, are given in table 1 . Both spectrometer systems are supplied with an image intensifier, which are tapered onto the respective cameras to increase the transmission to the chip. Each system contains of a CCD camera system allowing frame rates up to $30 \mathrm{fps}$ in full frame mode. In Section 3 the method is described to discriminate the background light from the actual active CXRS signal. Based on the beam modulation a scheme is developed for the acquisition of frames. The diagnostic beam is operated over 3s to 4s and a modulation frequency of $5 \mathrm{~Hz}$ with a 1:1 duty cycle. The frame rate of the camera is $10 \mathrm{~Hz}$ and synchronized with the frequency of the beam allowing the separate acquisition of the passive and sum of active and passive CXRS. 
The toroidal system, due to the generally higher rotation velocities, can tolerate larger absolute uncertainties with respect to rotation resolution, while keeping the same relative resolution. Two rows on the chip are combined (binned) in the direction of the chip correspoding to the radial direction in the plasma. This is done to increase the read out speed. A radial channel (fiber) is typically represented by 3(6) pixel $\times 256$ pixel, whith the carbon spectrum itself is only covering a small part in spectral direction allowing for the neon reference line $(\lambda=529.82 \mathrm{~nm})$ being displayed continuously.

The whole width of the chip covers a spectral range of $\sim 4 \mathrm{~nm}$ (table 1), which corresponds to temperature between $20 \mathrm{eV}$ and $3000 \mathrm{eV}$ (Typical $\mathcal{O}(100 \mathrm{eV})$ ) measured from the Doppler broadening. The spectral resolution of the spectrometer allows a

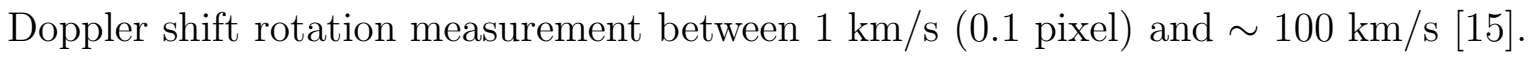
The situation for the poloidal spectrometer is more specialized to the needs of the measured parameters. The setup is comparable with the toroidal system with one distinct difference, due to the high spectral resolution of $(1.0799 \pm 0.0003) \mathrm{pm} /$ pixel only a limited spectral window can be obtained across the chip. The spectral window of $1 \mathrm{~nm}$ allows temperature measurements of up to $900 \mathrm{eV}$ with a resolution well in the range of $\mathrm{eV}$, taking into account only the dispersion of the spectrometer. The resolution in terms of Doppler shift measurements of the ion velocity is in the order of 0.3 pixel (statistical uncertainties), meaning $200 \mathrm{~m} / \mathrm{s}$ when considering the dispersion relation only. The radial binning, the combination of several CCD lines, is performed offline. Since the emissions are directly imaged onto the spectrometer and hence the camera chip one has complete freedom whether to analyze the whole frame line by line with a small intensity, or to choose a binning which offers more intensity. The system is able to obtain full frame images with a rate well above the beam modulation rate. The resolutions can be severely limited by the photon (Poisson) statistics which has to be taken into account for later measurements and accuracy discussions, as given in section4.

\section{Measurement Principles}

In this section the underlying measurement method is discussed. Remarks regarding correction and calibrations methods are given. In case of the presented diagnostic the Charge Exchange between Hydrogen $(\mathrm{H})$ and fully ionized carbon $(\mathrm{C})$ is used [17, 25].

$$
H+C^{6+} \rightarrow H^{+}+C^{5+}(n, l), C^{5+}(n, l) \rightarrow C^{5+}\left(n^{\prime}, l^{\prime}\right)+h \nu
$$

For the carbon spectroscopy considered in this work the visible spectral range is used and hence the most suitable transition $n \rightarrow n^{\prime}=8 \rightarrow 7$ at $529.1 \mathrm{~nm}$ [45] is selected. A least square minimization method is applied to find an appropriate gaussian function (fit) to the measured spectra. One of the most significant features of the charge exchange diagnostic at TEXTOR is the modulation of the utilized neutral beam. While most other CXRS diagnostics use separate viewing lines [10, 24] or complex fitting methods $[12,18,44]$ to subtract the CXRS and bremsstrahlung background from the active signal, the diagnostic presented uses frames with and without beam signal to aquire the active 
Figure 4: (ONLINE COLOR) (left) Intensity evolution of the CVI Line including the beam modulation (right) CXR Spectrum from the poloidal observation.

and passive parts of the spectrum, a method also applied at the DIII-D Tokamaks NBI injectors. The cameras are triggered following the beam modulation, collecting emissions from active and passive phases. The intensity evolution during one TEXTOR discharge, observed by both observation systems, is displayed in figure 4 . It shows a clear modulation which amounts to a poloidal modulation depth of $\Delta(I) / I=0.199 \pm 0.060$ and toroidal modulation depth of $\Delta(I) / I=0.054 \pm 0.027$, which are the typical values for a TEXTOR discharge with densities around $n_{e} \cong(2-3) * 10^{19} \mathrm{~m}^{-3}$. The smaller ratio for the toroidal view is caused by the longer line of sight background light integration. The intensity sufficient for highly time resolved measurements can not be reached. The overall modulation is about $20 \%$ but the quality of the measurement and the spectral shape is mostly governed by the total intensity acquired through the whole discharge. The typical time window used averaging is three seconds, giving 15 active and 15 passive frames (cf. figure 4). This means, there is a time resolution of $100 \mathrm{~ms}$ necessary to gather the necessary frames during the operation of the diagnostic beam, but the actual measurements are performed on a data set acquired over the whole beam period of three to four seconds for reasonably intensities. The two spectra are then used to obtain the active charge exchange spectra as shown in figure 4 . The active and the passive component of the spectra can be clearly discriminated. A gaussian fit is added two show the good agreement with the expected spectral shape assuming the ideal case of a single emission line. In the case displayed the poloidal observation system was used, allowing to substantiate on the reason for the modulated beam approach. In the case of the toroidal rotation typically measured with the NBI, the active CXRS spectrum is shifted from the passive background, and hence can be more easily discriminated by a multi component gauss fit. In case of small poloidal rotation this is not the case (figure 4), there is no large shift in the spectra. In the following paragraph a short description of the available parameters is given, the rotation velocity measurements as well as the measurement of $T_{i}$ and $n_{i}$ or intensity. Peak position, width and intensity based on the gaussian fit are used. The velocity is simply deduced via the Dopplershift $v_{i o n}=-c \cdot \frac{\lambda_{0}-\lambda}{\lambda_{0}}$, where $\mathrm{c}$ denotes the speed of light, $\lambda_{0}$ the rest frame wavelength and $\lambda$ the Doppler shifted $\lambda$. The ion temperature is given by spectral width of the observed emission line, assuming gaussian spectra [13]:

$$
T[e V]=\left(\frac{\lambda_{F W H M}}{\lambda_{0}}\right)^{2} \cdot \frac{m c^{2}}{8 e \cdot \ln (2)}=\left(\frac{\sigma_{s} \cdot D}{\lambda_{0}}\right)^{2} \cdot 1.68 \cdot 10^{8} \cdot \mu[a m u] .
$$

$\lambda_{F W H M}$ gives the width of the gaussian spectrum in terms of nm while the other terms are used as conversion factors. $\sigma s$ is the width in pixel and $D$ the dispersion. For the impurity density an iterative code like CHEAP $[42,44]$ is used to solve a set of differential 
equations based on the measured photon flux and the known beam components. The calculation of the carbon density is done in a self consistent way, taking beam attenuation and the carbon density interdependently into account

\subsection{Absolute Calibration for $v_{p o l}, v_{t o r}, n_{i}$}

The two observation systems utilize two distinct ways to determine the rest frame wavelength $\lambda_{0}$ necessary for the rotation (cf. section 3 ) as well as for the temperature (cf. eq.2) measurements. The poloidal system uses differential Doppler spectroscopy [20], while the toroidal system relies on a neon reference line, which is observed by one of the channels imaged onto the CCD chip. Differential Doppler spectroscopy utilizes two opposing lines of sight to acquire one red shifted and one blue shifted spectrum from the same rotation. In case of the poloidal observation system, this is one channel from the top of the top and one optical fiber channel from the bottom (cf figure 2). Via this setup one obtains two spectra with distinctly the same shift in opposite directions. Both represent the rotation velocity $\left(v_{p o l}\right)$ with respect to the rest frame. Both spectra are fitted and the positions of $\lambda_{\text {red }}$ and $\lambda_{b l u e}$ are then used to calculate the position of $\lambda_{0}$ on the CCD chip. The mean value of the peak position corresponds to the position of $\lambda_{0}$ on the CCD: $\operatorname{Pos}\left(\lambda_{0}\right)=0.5 *\left(\operatorname{Pos}\left(\lambda_{\text {red }}\right)+\operatorname{Pos}\left(\lambda_{\text {blue }}\right)\right)$. With sufficient photon statistics this method is accurate in the order of the velocity resolution of the spectrometer $(<1$ $\mathrm{km} / \mathrm{s}$ ) and hence the best method to assure a valid measurement of small $v_{\text {pol }}$. A similar system is used at the TJII stellarator [4]. In contrast to the common use of reference lines this method presents a direct measurement of the rest frame wavelength and is hence not prone to systematic errors, compensated here by the differential approach.The main systematic errors by using a reference line are angular deviations from misaligned mounting in front of the spectrometer, causing a strong shift in rotation velocity. The toroidal observation system uses a neon reference line imaged onto the chip which is continuously emitted during the whole discharge. The line $(529.82 \mathrm{~nm})$ is fitted similarly to the CXRS spectra and its position used to determine the position of the rest frame wavelength. Since all fibers, including the reference channel, are mounted collectively in front of the spectrometer, the angular discrepancy is negligible. An absolute photon flux calibration for the line emission intensity measured is needed for the density calculations, it is performed using an integrating sphere in front of the observation system at the position of the neutral heating beam. This allows to measure the ratio between emitted photon flux and the counts on chip. This is then input for the CHEAP code [42, 44].

\subsection{Radial Calibration}

There are two options for the radial calibration, the first option is to illuminate the optical fibers or the spectrometer slit, imagining them onto the position of the hydrogen beam inside of the vessel. Hence establishing a direct relation between the fiber or slit position and the radial position. This method is very accurate and typically used during opening of TEXTOR. Since a vessel opening is only performed once every year a second 
reliable option is needed. This option is based on the Doppler shift spectroscopy using Beam Emission Spectroscopy (BES). This method is commonly applied and results in Doppler shift spectra for each radial position, from which the radial position is deduced.

\section{Accuracies}

In this next section the accuracy and the applied uncertainty calculations for the toroidal and poloidal impurity velocity as well as for the the ion temperature are given. Corrections regarding finite slit height[29], Zeeman effect and Fine-structure are applied $[2,21,22]$ during the analysis process and thus accounted for regarding the resulting temperatures and velocities. Corrections regarding CXRS cross-sections are negligible due to the low temperatures and perpendicular viewing angle [43].

The measurement uncertainties in rotation and $T_{i}$ are calculated based on the uncertainty of the dispersion and the statistical uncertainty on width and position of the gaussian fit. Some of the numbers regarding the resolution have been discussed earlier (Section 2.3); with the dispersion given in table 1 the poloidal system is capable of resolving $1 \mathrm{~km} / \mathrm{s}$ rotation (1.6 pixel on the CCD) while $100 \mathrm{eV}$ in $T_{i}$ are equivalent to a FWHM of 118 pixel. For the toroidal system $1 \mathrm{~km} / \mathrm{s}$ means 0.1 pixel and $100 \mathrm{eV}$ are $\sim 7$ pixel. This, with respect to the measurement accuracy, means the following: First the poloidal system is well equipped for rotation and $T_{i}$ measurements, but due to limitations in the intensity, is prone to errors due to poisson statistics. The smaller the intensity the more distorted the spectral shape can be influencing e.g. the subsequent position calculation. With respect to the measured quantities this means a direct impact on the accuracy. Intensity and dispersion are intertwined and both need to be optimized together in order to obtain reasonable results. High dispersion requires high intensity since less photons are accessible per pixel the higher the dispersion is set. Since we require highly accurate velocity measurements this can be quite critical, while $T_{i}$ is well defined within the required accuracies. For the toroidal system the situation is less

Figure 5: (ONLINE COLOR) $T_{i}$ measured by poloidal and toroidal observation

complex, first because of the smaller dispersion and less dominance due poisson statistics and second due to the different scale $(>10 \mathrm{~km} / \mathrm{s})$. The $T_{i}$ measurement on the other hand is more difficult due to the dispersion. In figure 5 a temperature measurement is shown, comparing the poloidal and toroidal data. The agreement between both is reasonably well considering both the errorbars for $T_{i}$ and the radial resolution(cf. 3). This plot shows the different capabilities of the two systems and clearly shows why the poloidal system is preferred for $T_{i}(1 \mathrm{eV} \widehat{=} 1 \mathrm{pixel})$. The uncertainties are:

$$
\begin{aligned}
& \sigma_{v}=c \cdot \sqrt{\Delta_{p}^{2} \cdot \sigma_{D}^{2}+D^{2} \cdot\left(\sigma_{r p}^{2}+\sigma_{p}^{2}\right)} / \lambda_{0} \\
& \sigma_{T}=T_{i} \cdot\left(2 \sqrt{\left(\sigma_{D} / D\right)^{2}+\cdot\left(\sigma_{s} / \sigma_{\text {spec }}\right)^{2}}\right)
\end{aligned}
$$


(3) describes the calculation of the uncertainty for the velocity, taking into account the Doppler shift in terms of chip position $\left(\Delta_{p}[\right.$ pixel $\left.]\right)$, the dispersion $(\mathrm{D}[\AA /$ pixel $])$ and its uncertainty $\left(\sigma_{D}[\AA /\right.$ / pixel $\left.]\right)$ as well as the uncertainty on the peak position $\left(\sigma_{p}[\right.$ pixel $\left.]\right)$ and the reference position $\left(\sigma_{r p}[\right.$ pixel $]$ ) (both on the chip). The gaussian fit uses as input parameters the obtained spectral data and statistical information from the data set. For the $T_{i}$ a similar calculation (eq.4) is performed. The dispersion as well as its uncertainty are included into the calculation as well as the spectral width $\left(\sigma_{\text {spec }}[\right.$ pixel $\left.]\right)$ and its uncertainty $\left(\sigma_{s}[\right.$ pixel $\left.]\right)$. From this one can deduce the typical error bars, which amount for $v_{\text {pol }}$ typically to $\sigma=0.7 \mathrm{~km} / \mathrm{s}$ and for $v_{\text {tor }} \sigma=5 \mathrm{~km} / \mathrm{s}$. For $T_{i}$ it is deducible from (4) that the uncertainty scales with the temperature itself, and thus is larger in the plasma core than in the edge. The errorbar for the poloidaly measured $T_{i}$ lies between $2 \mathrm{eV}$ and $10 \mathrm{eV}$, while the toroidally measured $T_{i}$ has an uncertainty of 5 to $40 \mathrm{eV}$.

The present status of the diagnostic utilizes the direct imaging in order to supply as much intensity to the high dispersion spectrometer as possible. The poloidal diagnostic produces very good results with respect to the relative accuracy $(0.7 \mathrm{~km} / \mathrm{s})$ which depends strongly on the intensity supplied by the observation system on top of the tokamak vessel. The reference channel (cf. figure 2)however is still transferred via fiber and those signals still suffer from small intensities. Since the spectrometer components are not in any way influenced or moved during the measurement campaign, it is assumed that the reference position is not changing from discharge to discharge. The setup is mounted without connection to the torus, thus being unaffected from vibrations. The temperature of the spectrometer as well as the camera setup is at a constant level. As mentioned above effects of e.g. magnetic fields are taken into account when performing the analysis. This allows to calculate an average reference position which is well described. The uncertainty for the average value is well below one pixel $(2.41 \mathrm{pixel} / \sqrt{26}=0.47 \rightarrow 0.2 \mathrm{~km} / \mathrm{s})$ and small compared to the uncertainty of $0.7 \mathrm{~km} / \mathrm{s}$. Regarding the CCD and Image-intensifiers used no significant influence on the accuracy is seen or expected. The quantum efficiency of CCD and MCP are here the most significant component since impacting on the actually available intensity. However, the small original signal requires intensification via a Multi Channel Plate(MCP) thus even the $10 \% \mathrm{QE}$ are acceptable and within industry norm. Regarding readout time and noise it can be said that compared to the $100 \mathrm{~ms}$ frame integration the read out time is negligible and the noise is well below the poisson levels observed. The Taper connecting MCP and CCD has a structure of $6 \mathrm{~m}$ and thus does not effect the resolution.

\section{Data \& First Results}

An example is shown to demonstrate the capabilities of the new CXRS diagnostic with respect to the two rotational directions as well as $T_{i}$ and the $E_{r}$ measurement. The capability of TEXTOR is used adapting the toroidal plasma rotation via the net torque input from the co-current and a counter current Neutral Beam Injectors (figure 1). A series of discharges with constant input power of 1.3 MW from, a toroidal field of 2.1 
$\mathrm{T}$ and a plasma current of $410 \mathrm{kA}$ i shown. In the course of the different discharges the momentum fraction between the two tangential beams is gradually changed from full counter current $(+)(\# 106846)$ via balanced beam (\#106848) to full co current (-) (\#106850) injection to study the behavior of plasma impurity (CVI) rotation and the measurement procedure for $v_{p o l}$ and $v_{t o r}$. The TEXTOR plasma is centered around $1.75 \mathrm{~m}$ of the major radius with a minor radius of $r=0.46 \mathrm{~m}$, locating the last closed flux surface at $R \sim 2.21 \mathrm{~m}$. The first set of data presented in figure 6 shows the

Figure 6: (ONLINE COLOR) $v_{t}$ or and $v_{\text {pol }}$ during a momentum input scan, changing the fraction between the two beam injectors, keeping the input power constant.

influence of momentum input on the toroidal rotation in TEXTOR. It is visible that the momentum input from the neutral beam accelerates the center of the plasma, causing a co- or counter-rotation, while it is also visible that even with balanced beam injection (similar for ohmic discharges) a rotation in counter direction is observed. The changes in momentum input can be observed across the whole profile except the SOL area, where the profiles are underlying a general flow into the counter direction. The profiles are fixed at the edge where the rotation is slowed down due to friction with neutral particles. The profile structure can be explained with the perpendicular viscosity, keeping the outer part connected to the innermost momentum source. The poloidal rotation shown in figure 6 shows profiles which, towards the center $(\mathrm{r} / \mathrm{a}=0.5)$, follow the trend of increased momentum. While the momentum input might explain those changes, the most striking observations are in the outer plasma edge: the rotation changes from the electron diamagnetic drift direction $($ counter + ) to the ion diamagnetic drift direction (co -). Only the discharges with dominant NBI 2 injection show the strong increase in edge rotation, thus a connection with the different confinement of fast ions for the two beams can be assumed. The gradients for $T_{i}$ in this area are comparable (figure 7 ) and seem not to drive the changes. Figure 7 shows $T_{i}$ for all discharges discussed above. The $T_{i}$ is quite similar due to constand NBI power if taking into account both the radial resolution as well as the temperature uncertainty. It has be estimated from calculations [32] that about $8 \%$ of the beam ions are lost via orbit losses when using NBI 2. Comparing this quantitatively with calculation is beyond the scope of this paper. This example once more stresses the value of a high resolution diagnostic focussing on the poloidal velocity resolution. The last result presented here is the $E_{r}$ deduced according to the radial

Figure 7: (ONLINE COLOR) $T_{i}$ acquired with the poloidal system during a momentum scan, changing fraction between the two neutral beam injectors at constant input power.

force balance equation $[7,34]$ given in eq.5. It is seen from (5), that the $E_{r}$ follows the contributions from $v_{t o r}$ and $v_{p o l}$, dominated by $v_{p o l}$ (cf. figures 6 and 8).

$$
E_{r}=E_{r(\text { diam. })}+E_{r(\text { pol })}+E_{r(t o r .)}=+\frac{1}{Z_{i} e n_{i}} \frac{d p_{i}}{d r}-v_{p o l} B_{T}+v_{t o r} B_{p}
$$


$p_{i}$ is the pressure gradient and $n_{i}$ is the ion density. $Z_{i}$ is accounting for the impurity charge and $B_{p, T}$ are the respective components of the magnetic field. For circular shaped

Figure 8: (ONLINE COLOR) $E_{r}$ during a momentum input scan, changing the momentum fraction between the two neutral beam injectors (constant input power).

L-Mode limiter plasmas at TEXTOR with low resistivity it has been shown that the diamagnetic term can be neglected [1] as found for other L-Mode plasmas [24].

\section{Conclusion}

In the course of this paper we have presented the latest status of the CXRS diagnostic on the RuDI Hydrogen Diagnostic Beam at TEXTOR. A poloidal and toroidal diagnostic is successfully integrated into the TEXTOR environment. The performance is dominated by the line intensity which can be observed.

The toroidal rotation can be measured with an accuracies of $5 \mathrm{~km} / \mathrm{s}$ and the poloidal rotation is resolved well below $1 \mathrm{~km} / \mathrm{s}(0.7 \mathrm{~km} / \mathrm{s})$. The diagnostics deliver a spatially resolved measurement $\sim 1 \mathrm{~cm}$ of the $E_{r}$ with a resolution of $\sim 2-5 \mathrm{kV} / \mathrm{m}$. This is achieved by combining the high spectral resolution of the poloidal spectrometer system, with a dispersion of $(1.0799 \pm 0.0003) \mathrm{pm} /$ pixel and the toroidal system delivering a dispersion of $1.624 \pm 0.005 \mathrm{~nm} /$ pixel. The data presented is a good example of the capabilities of this diagnostic, showing changes of rotation from $-50 \mathrm{~km} / \mathrm{s}$ up to $100 \mathrm{~km} / \mathrm{s}$ for the toroidal system as well as changes from $-3 \mathrm{~km} / \mathrm{s}$ up to $5 \mathrm{~km} / \mathrm{s}$ for the poloidal rotation. $T_{i}$ is measured almost up to $1000 \mathrm{eV}$ and $E_{r}$ can be calculated over the whole domain accessible for this diagnostic. The system can be regularly used at TEXTOR to measure impurity (carbon) rotation and temperature on a discharge by discharge basis, meaning an integration time of about 3-4 s is needed. A method is established to consistently measure the radial electric field at one toroidal position, deriving it directly from the measured components. By using a diagnostic beam CXRS measurements are in principal feasible under all plasma conditions. Compared to other diagnostics like Doppler Reflectometry [6] or the heavy ion beam probe [24] the advantages of this CXRS diagnostic is its high spectral and rotation resolution as well as its large coverage over half of the minor radius $0.5<r / a<1.1$ including the whole plasma edge. This diagnostics supplies all necessary information for the radial electric field calculations and the radial force balance. No additional input parameters are needed $[1,5]$ the rotation as well as the pressure gradient are measured.

In order to gain more access to low intensity scenarios further improvements to the setup up are performed including actively cooled camera system removing the background noise and allowing operation during scenarios with higher radiation levels. 


\section{Acknowledgments}

This work has been performed with strong support from G. Herpers, K. von Bovert, K. Klören and D. Schüller for the technical aspects. Results are part of a finished $\mathrm{PhD}$ thesis at the "Heinrich-Heine-Universität, Düsseldorf". This work was partially supported by the German Research Foundation (DFG) under grant No. UN 265/1-1 and the Research Training Group GRK 1203 - Dynamics of hot plasmas.

[1] Coenen et al. Volume 32D of ECA, 2008.

[2] Ch. Busch. Volume 50 Energy Technology Edition, FZJ, 2006. ISBN 3-89336-433-1.

[3] E Busche et al. Plasma Physics and Controlled Fusion, 39(9):1327-1338, 1997.

[4] J. M. Carmona et al. Review Of Scientific Instruments, 77(10):10F107, 2006.

[5] J. W. Coenen. Volume 39 of Energy 83 Environment. FZJ, 2009. ISBN :978-3-89336-574-6

[6] G. D. Conway et al. Plasma Physics And Controlled Fusion, 46(6):951-970, 2004.

[7] J. Cornelis et al. Nuclear Fusion, 34(2):171-183, 1994.

[8] M. F. M. De Bock et al. Nuclear Fusion, 48(1):015007 (16pp), 2008.

[9] P. P. Deichuli and G. I. Shulzhenko. Fusion Science And Technology, 47(1T):330-332, 2005.

[10] D. J. Den Hartog et al. Review Of Scientific Instruments, 77(10):10F122, 2006.

[11] B. P. Duval et al. Plasma Physics And Controlled Fusion, 49:B195-B209, 2007.

[12] Bishop et al. Plasma Physics And Controlled Fusion, 35(6):765-773, 1993.

[13] Bogen et al. Journal Of Nuclear Materials, 220:472-477, 1995.

[14] Burrell et al. Review of Scientific Instruments, 75(10):3455-3457, 2004.

[15] Finken. Physical Review Letters, 94(1):015003, 2005.

[16] K. H. Finken et al. Nuclear Fusion, 39(5):637-662, 1999.

[17] R. J. Fonck et al. Physical Review A, 29(6):3288-3309, 1984.

[18] S. Gangadhara et al. Review Of Scientific Instruments, 77(10):10F109, 2006.

[19] H. Geissler. Geissler tube. Encyclopedia Britannica Online, 2008.

[20] N.C. Hawkes and N. Peacock. Review of Scientific Instruments, 63(11):5164, 1992.

[21] J. D. Hey et al. Contributions To Plasma Physics, 34(6):725-747, 1994.

[22] J.D. Hey et al. In Proc. 20th EPS Conference, Volume 17C, pages 1111, Part III, Lisbon, 1993.

[23] A. Huber et al. Plasma Physics And Controlled Fusion, 42(5):569-578, 2000.

[24] K. Ida. Plasma Physics And Controlled Fusion, 40(8):1429-1488, August 1998.

[25] R. C. Isler. Plasma Physics And Controlled Fusion, 36(2):171-208, 1994.

[26] A. A. Ivanov et al. Review Of Scientific Instruments, 75(5):1822-1825, 2004.

[27] Alexander N. Karpushov et al. Fusion Engineering and Design, 66-68:899-904, September 2003.

[28] A. Kreter. Volume Juel-3860 of Berichte des Forschungszentrum Juelich 2001.

[29] A. Last and J Mohr. Wissenschaftliche Berichte FZKA, 6885:31, 2003.

[30] A. A. Listopad and J. W. Coenen et al. volume 81, page 02B104. AIP, 2010.

[31] NIST. Nist atomic spectra database.

[32] J. P. H. E. Ongena et al. Fusion Science And Technology, 53(2T):367-376, 2008.

[33] Pluecker. Annalen der Physik und Chemie, 103:88, 1858.

[34] V. Rozhansky and M. Tendler. Reviews of Plasma Physics, 19:147, 1996.

[35] V. A. Rozhansky et al. Plasma Physics Reports, 27(3):205-210, 2001.

[36] O. Schmitz et al. Journal Of Nuclear Materials, 363:680-685, June 2007.

[37] O. Schmitz et al. Nuclear Fusion, 48(2):024009, 2008.

[38] R. P. Schorn et al. Nuclear Fusion, 32(3):351-359, 1992.

[39] R. P. Seraydarian et al. Review of Scientific Instruments, 57(2):155-163, 1986.

[40] D. Testa et al. Nuclear Fusion, 46(5):562-579, May 2006.

[41] Jaspers R, M. von Hellermann page pp. 205. Plenum Press (New York \& London), 2002.

[42] M. von Hellermann. JET Internal Page, Exp.Department, Core Spectroscopy Group, 2000. 
[43] M. von Hellermann et al. Plasma Physics And Controlled Fusion, 37(2):71-94, 1995.

[44] M. G. von Hellermann et al. Burning Plasma Diagnostics, 988:165-176, 2008.

[45] W.L. Wiese et al. Journal Of Physical And Chemical Reference Data. 1996. 


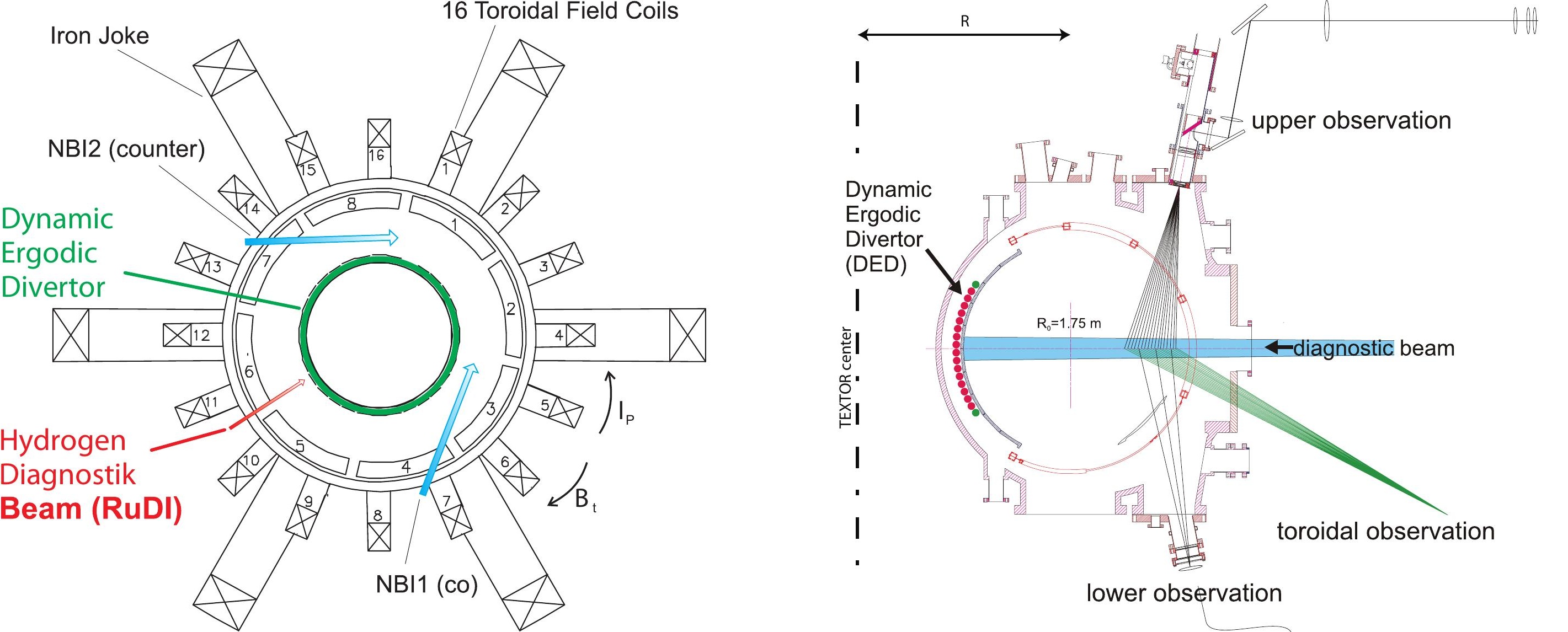




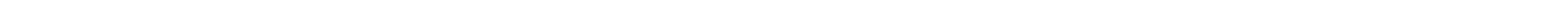



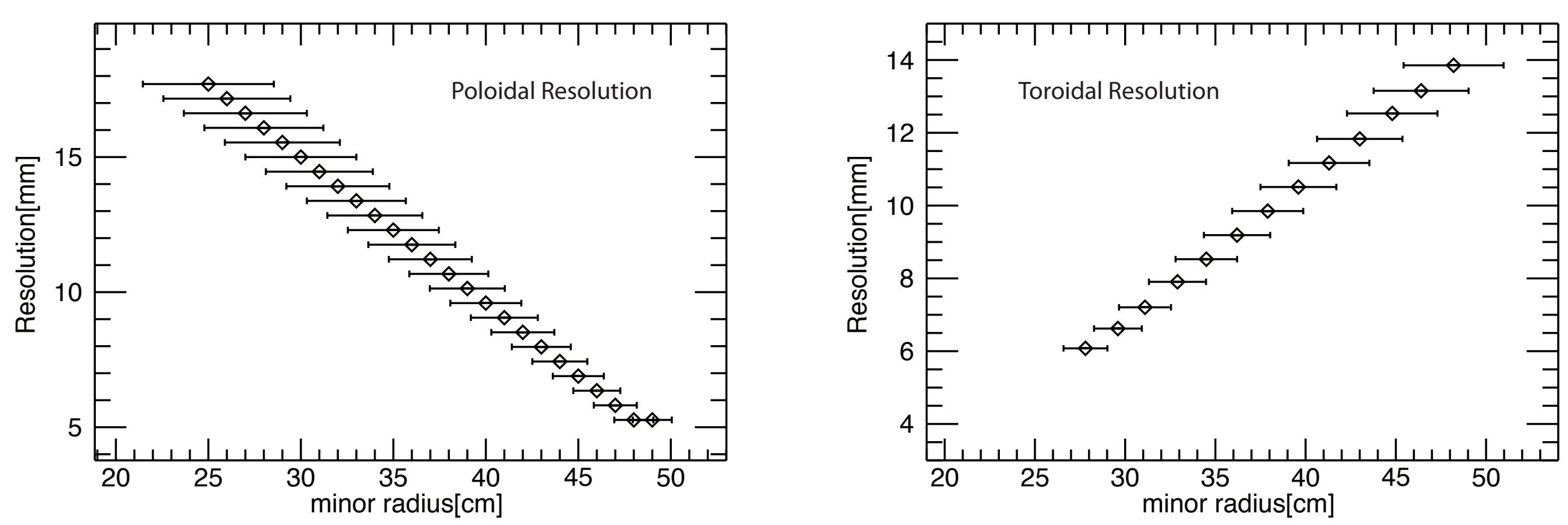
CVI Intensity
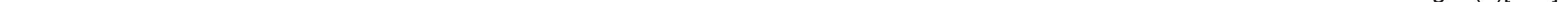


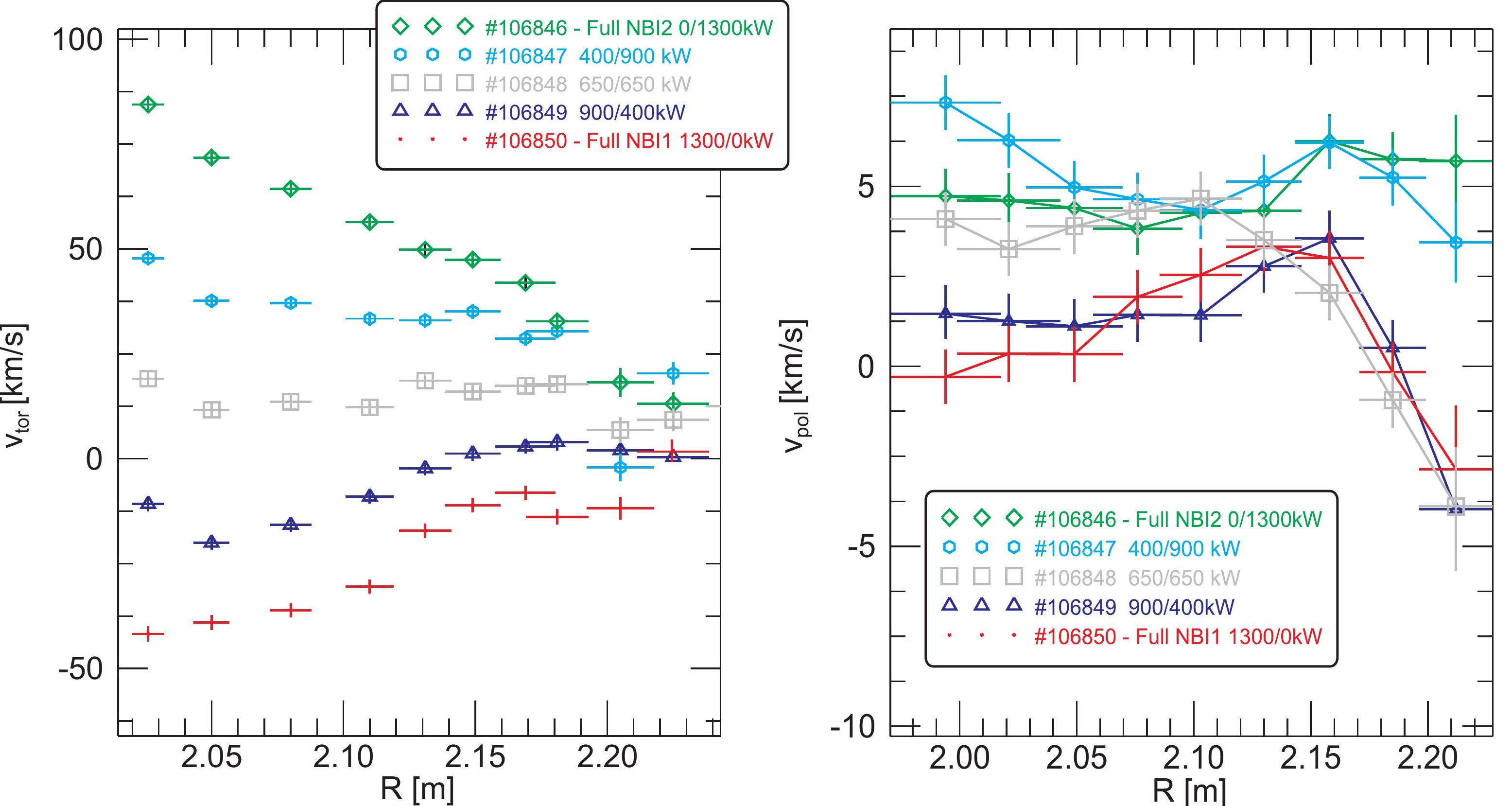




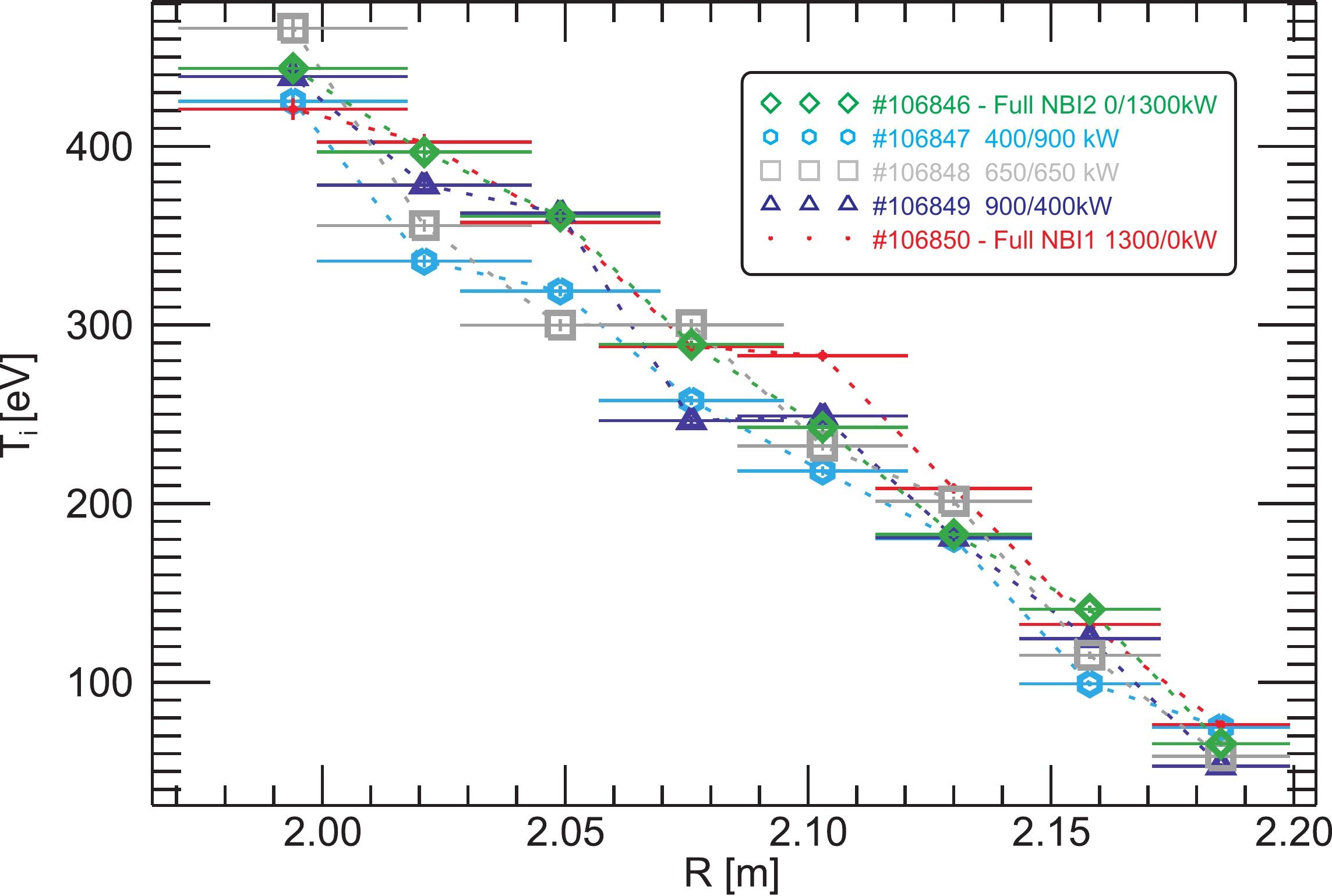




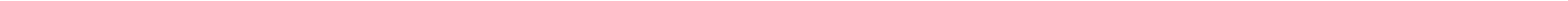

